Journal of Finance Research

\title{
Research on the Future Trend of New Retail and E-commerce
}

\section{Bingxi Li*}

Graduate School of International Cooperation Studies, Takushoku University, Tokyo, 112-8585, Japan

\begin{tabular}{l}
\hline ARTICLE INFO \\
\hline Article history \\
Received: 14 July 2020 \\
Revised: 21 July 2020 \\
Accepted: 9 October 2020 \\
Published Online: 16 October 2020 \\
\hline Keywords: \\
New retail \\
E-commerce \\
Future development \\
Trends
\end{tabular}

\section{Introduction}

$\mathrm{I}$ $\mathrm{n}$ the rapid development of information technology and Internet technology, China's big data technology, artificial intelligence and block chain technology innovation speed is very fast. For online transactions based on the network environment, it has promoted the innovation and development of new business forms and trading methods. Although in the past period of time, the network, that is, online platform, has developed very fast, the integration of the construction platform and offline platform is the mainstream and development trend of the future. Through the understanding of the creators of many online shopping platforms, the combination of online and offline is also the mainstream of future development, and a lot of energy has been invested in the work of combining online and offline.

\section{ABSTRACT}

In the rapid development of information technology, the term new retail was derived based on online shopping, and as the creator of largest online shopping platform in China, Ma Yun, made a related concept discussion on new retail, which caused a great response in the whole society. Therefore, this paper will study the future development trend of the new retail model, as well as the controversy existing in the new retail itself and many successful cases. Through analysis and discussion, the paper will discuss the future development of new retail and e-commerce trends.

\section{New Retail Overview}

\subsection{New Retail Concept}

The concept of new retail is put forward by Ma Yun at the Yunqi Conference held in October 2016, that is, in the future shopping process, the retail model will gradually change to a combination of online and offline, while logistics provides assistance to achieve new retail. The combination of the three can achieve a real new retail. Because in the past development process, online platform construction and sales model have had a strong impact on the traditional offline sales, and the current trend of combining online and offline has made the retail industry transformation work in the entire Internet era with a specific direction, so that the whole Internet era of retail reform work has a specific direction, it is also feasible. The characteristics of the new retail is the combination of online and offline. In the process of gradual

*Corresponding Author:

Bingxi Li,

Correspondence address: Graduate School of International Cooperation Studies, Takushoku University, Tokyo, 112-8585, Japan; E-mail:lbxtzdx@yeah.net. 
integration, there is a fusion state in which you have me and I have you ${ }^{[1]}$.

\subsection{Breakthrough in Physical Retail}

Since the emergence of online shopping platforms, the development of the real retail industry in China can be described as mixed. On the one hand, the number of closed stores such as major department stores and some shopping malls has greatly increased, while the performance of daily operations has continued to decline. On the other hand, some industries are constantly seeking to transform and upgrade under the impact of new fields and new models. Especially with the new retail concept put forward by Ma Yunqi at the 2016 Yunqi conference, both physical retail and online retail are beginning to think about whether or not, as Ma Yun said, e-commerce will form the mode of online and offline integration with logistics in the coming decades. ${ }^{[2]}$.

In a survey of traditional retail sales data in 2016, it was found that layoffs and closures were frequent in supermarkets and department stores. In related reports issued by third parties, $67 \%$ of department stores and supermarkets showed a decline in both company revenue and net profit in the first half of 2016. And the development of this phenomenon has not been significantly improved so far, so it can be considered that the current online growth is weak, and offline need online data to transform its sales model, so the organic integration between the two will be a very favorable measure, which will have a very positive impact on the retail industry. The main thing is that the current technological innovation, which makes the realization of new retailing more reliable, supports the rapid development of the Internet and the rapid progress of information technology and intelligent technology, making the application of scientific and technological means more and more extensive in the retail industry ${ }^{[3]}$.

\subsection{The Real Online and Offline Integration Has Not Yet Been Achieved}

Although the combination of online and offline is an inevitable trend for the retail industry, and has been promoted gradually, but based on the current social development background and technology development background, although many e-commerce giants have carried out layouts and made some progress, there still have not formed a real integration between online and offline. For traditional stores and supermarkets, they already have a large and systematic team and operation model, in the process of integrating the online sales model into the of- fline sales system, there is a great contradiction between them, and there are also conflicts of interest. So through the new way of thinking to carry out the operation of offline supermarkets, although it is innovative, the actual implementation is not satisfactory. Especially in the process of offline expansion, because of the need to establish new stores and recruit new talents and teams to operate, there are great difficulties in the process of site decoration and talent selection.

For e-commerce brands, in the process of seeking online and offline integration, the most choice is to open offline stores. Taking many hot brands on Taobao as an example, the three squirrels, membrane family and $\mathrm{Yu} \mathrm{Ni}$ Fang, etc., in addition to selling on the online platform, also set up offline physical stores in many crowded cities and regions. Because of the traffic and visibility provided by the online platform, the gains from the offline physical stores opened are very significant. In addition to Taobao, other network platforms, including JingDong, Dangdang and Jumei, etc., some powerful and hot-selling brands have already carried out similar physical store layout offline. For these online hot selling brands, their own popularity is relatively high, so there is no need to worry about the flow problem in actual sales, but at the same time, the same problems as the traditional retail industry are the location of the decoration and the choice of supply chain. There are many online hot selling brands on the opening of physical stores have encountered these problems.

\subsection{Reflections on New Retail and Traditional Retail}

From the perspective of traditional retailers, although new retail has brought impact and influence to the traditional retail industry, it also means that the value reconstruction of the traditional retail industry has come. After analysis by some Chinese chain experts, it was concluded that in the future development process, traditional retail will move towards "big" and "small" -- twopole direction. The so-called "big" refers to large-scale shopping mall that combines entertainment, leisure and consumption. This conclusion is not unfounded, because there are nearly 1,000 large and medium-sized commercial projects in China's current large and medium-sized cities, including Big-name supermarkets such as Wanda square and Outlets. The "small" refers to convenience stores, because the consumption of people with credit is constantly upgrading, and the urban residents of the demographic changes, so the demand for convenience has become more and more prominent. For the new after-sales industry, based on convenience stores to expand 
and cover the new retail industry is a very important model. JD President Qiangdong Liu, in his speech and planning for corporate strategy, announced that within the next five years JD would open more than 1 million convenience stores in Chinese cities.

Thinking about the future development from the perspective of new retail, the most important change in the development process is to optimize the service. Because compared to the traditional retail industry, services in the online sales process are mainly reflected in pre-sales and after-sales, and lack of intermediate communication links, so in the process of shifting to offline sales, more optimization of service content and service methods is required. In combination with the actual needs of consumers, as well as consumer pain points in the process of consumption, when these problems are solved, the value of the new retail industry will increase, and for the development of the entire retail industry, real changes will occur.

\section{Future Trends in the New Retail Industry}

The future development trends of the new retail industry are mainly: the importance of supply chain protruding, the number of consumers based on membership system growth, the seamless connection between online and offline, and the rapid development of home decoration and beauty makeup industry.

\subsection{The Importance of the Supply Chain Is High- lighted}

Because in the early development process, the retail industry has not been greatly impacted and affected, so the attention of retailers themselves to the supply chain is the supply chain cost in the production cycle. And the impact of the new retail industry on the supply chain has gradually shifted to the agility of the supply chain itself. Because for the new retail industry that combines online and offline, the higher the agility of the supply chain itself, then compared with similar products and competitors themselves, the advantage position in the process of product sales and marketing will be higher. It is more beneficial to the development of the enterprise itself under the fierce competition environment. Therefore, in the future development of the new retail industry, to a large extent, the supply chain itself agility issues will be paid more and more attention.

\subsection{Increase in the Number of Consumers Based on the Membership System}

Because compared to individual customers, member customers belong to a fixed consumer group for offline physical stores. In the actual consumption process, the frequency of coming to the store is higher, and the actual expenditure of multiple consumption will also be more. Most of the profits of the physical store itself come from these members. For example, under the impact of the new retail industry, Macy's has constructed a new membership system for the entire department store. Some members with very high consumption will have 5\% cashback after the consumption limit reaches a certain upper limit on the same day. Through this new construction of the membership system to stimulate consumption, Macy's membership accounted for $50 \%$ of the company's total revenue after the system rectification, and the number of members in the class is also increasing. Therefore, for the new retail industry based on the combination of online and offline, many non-member customers in the past need to be converted into member customers, even if this part of converted customers is only $1 \%$ of non-member customers, but The profit brought by it is unmatched by those $99 \%$ of non-member customers

\subsection{Seamless Online and Offline Connections}

Since online and offline began to combine and influence each other, consumers themselves in the process of consumption more and more like to place orders online and take orders offline. For the retailer itself, this kind of purchase method also reduces the cost input in the distribution process, and also enables consumers to enter the store for consultation and consumption, and the traffic of the physical store becomes greater. With the passage of time, online orders and offline pick-up shopping method have become the choice of more and more people.

\subsection{The Rapid Development of Home Improve- ment and Beauty Industry}

For many retailers, after the emergence of online and offline integration of the new retail industry, through the comparison and research of the data, it is found that the sales of the two categories of Home improvement and Beauty under the impact of the new retail industry are much higher than the pure offline sales development level before. In the traditional retail industry, clothing is a well-deserved sales hegemony, and in the new retail industry model, home improvement and beauty developing faster than clothing. Home improvement services include not only traditional home decoration, but also pet supplies and garden supplies, which are in high demand today, are included in the content of home improvement 
services. And in the future, as people continue to improve the quality of life, the speed of home improvement services will continue to increase. The same is true of the beauty industry, people's pursuit of beauty and their own quality of life requirements will become higher and higher in the process of continuous development, so people will invest more and more in life, dress and beauty

\section{Conclusion}

To sum up, the author makes a comprehensive analysis of new retail in this paper, hoping to give readers some inspiration. For the development of e-commerce and new retail, with the improvement of people's living standards and the pursuit of life in the future, the consumption concept and quality of daily life will be improved. Therefore, the new retail industry derived from the combination of online and offline will receive more and more attention in the future, and with the rapid development of information technology and Internet technology, the relationship between e-commerce and the new retail industry will become closer and closer, which will bring about great changes for the entire sales industry and online shopping platforms.

\section{References}

[1] Hong Tao. "New Retail" and Future Trends in E-commerce $[\mathrm{J}]$. Business Economics Research,2017(006):64-70.

[2] Chen Lei. Current situation and trend of e-commerce under new retail mode $[\mathrm{J}]$.Mall Modernization ,2019 (012):56-57.

[3] Xu Haodong. Analysis on the Future Relationship Trend between "New Retail" and E-commerce [J]. Managers ,2017(27):286-287. 